\title{
Vier-Säulenmodell FO(U)R GW: Flexible, individuelle, kriterien- und kompetenzorientierte Leistungsbeurteilung
}

\author{
•kollerpk12@gmail.com, Institut für Geographie und Regionalforschung, Universität Wien \\ eingereicht am: 17.05.2018, akzeptiert am: 21.09.2018
}

\begin{abstract}
Der vorliegende Beitrag widmet sich einer der Kernkompetenzen für Lehrpersonen: der Leistungsbeurteilung. Dabei wird ein flexibles und lebensphasenbezogenes Konzept vorgeschlagen, das auf den der Leistungsbeurteilungsverordnung (LBV) bekannten Leistungsarten fußt und auf vier gleichgewichteten Säulen beruht: auf praktischen, grafischen, schriftlichen und mündlichen Leistungen.

Ein Mangel an dokumentierten Leistungen in einer Säule kann mit hervorragenden Leistungen in einer anderen Säule kompensiert werden, um individuelle Stärken von Schüler/innen bezüglich der Art der Leistungserbringung voll zur Geltung bringen zu können.

Die theoretische Umsetzung des Modells, welches seitens der Lehrperson ein entsprechendes Ausmaß an Methodenvielfalt und Organisationsfähigkeit erfordert, wird anhand des Lehrplans der 7. Klasse AHS illustriert. Es bleibt zu hoffen, dass das vorliegende Modell auch in der Praxis erprobt und im Zuge dessen auf Basis des Grundgedankens weiterentwickelt wird.
\end{abstract}

Keywords: Leistungsbeurteilung, Leistung, Leistungsarten, Kompetenz, Kompetenzorientierung

\section{Getting on track of the performances}

This article is dedicated to one of the core competences for teachers: assessment. It proposes a flexible and life-based approach which builds on the types of performances stated in the official Austrian Performance Assessment Regulation (LBV), and is based on four equally weighted pillars: practical, graphical, written, and oral performance.

With regard to an individual students' strengths in terms of performance fulfilment, a lack of recorded assessment in one pillar can be compensated by an outstanding performance in another pillar.

The theoretical implementation of the model, which requires a corresponding amount of methodological variety and organizational skills on behalf of the teacher, is illustrated with the example of the curriculum of the $7^{\text {th }}$ grade AHS (grammar school, upper form). It is much to be hoped that this model will also be tested in practice and, in the course of this, will be further developed on the basis of its fundamental idea.

Keywords: assessment, performance, performance types, competences, skills

\section{Idee und Grundriss des Modells}

Der vorliegende Beitrag widmet sich einer der Kernherausforderungen für Lehrpersonen: der Leistungsbeurteilung. Ein neues Konzept zur Durchführung derselben wird vorgeschlagen. Doch was bedeutet das Wort ,Leistung' eigentlich? Seiner ursprünglichen Herkunft (gotisch: ,laists' bzw. angelsächsisch: ,last') nach, kann man es mit „einer Spur nachgehen“ bzw. „jemandem/n folgen/nachgehen/begleiten " umschreiben (Verheyen 2018: 171).
In gewisser Weise also agiert die Lehrperson als ,Detektiv/in'. Was aber zeichnet einen guten Detektiv, wie etwa den berühmt-berüchtigten Inspektor Columbo, aus? Akribie, das aufwendige, arbeitsintensive Zusammentragen von kleinen Details und Indizien durch aktive Herbeiführung bzw. Kreation bestimmter Situationen und Settings. Schließlich ergibt eine Summe von Indizien ein schlüssiges Gesamtbild, welches den Inspektor auf die entscheidende Fährte führt. In gewisser Weise ist diese Akribie auch für eine Lehrperson nötig: Um die Leistung von Schüler/in- 
nen möglichst holistisch erfassen und schließlich beurteilen zu können, muss sie während eines Semesters möglichst vielfältige (methodische und soziale) Situationen bzw. Settings herstellen, um dort den Aktivitäten und Handlungen der Schüler/innen im jeweiligen Kontext ,nachspüren' zu können. Es benötigt Akribie bei der Entwicklung eines Beurteilungssystems, das einen möglichst breiten Raum für ,Entwicklungsspuren' bzw. ,Entwicklungswege' der Schüler/innen bietet. Denn je vielfältiger die Spuren einer Person sind, denen man nachgehen kann, desto detaillierter wird das Gesamtbild vom Vermögen und den Kapazitäten einer Person, welches sich erschließt. Je detaillierter wiederum Gesamtbilder ,erspürt' werden, desto fein kalibrierter und adäquater kann folglich die Leistungsbeurteilung durchgeführt werden.

Werfen wir zunächst einen Blick auf die gesetzlich genannten Arten von Leistung: die Leistungsbeurteilungsverordnung (LBV idF. BGBl. II Nr. 424/2016) kennt vier unterschiedliche Formen der Leistungserbringung bzw. -feststellung: mündliche, schriftliche, praktische und grafische Leistungen sind vorgesehen ( $\$ 3$ Abs. 1). Auch die Mitarbeit fußt gemäß $\$ 4$ Abs. 1 der Verordnung auf diesen vier Arten der Leistungserbringung.

Im Unterrichtsfach ,Geographie und Wirtschaftskunde' lässt sich mit diesen vier unterschiedlichen Leistungsformen sehr gut arbeiten, können doch praktische Leistungen z. B. im Rahmen von Exkursionen, bei der Durchführung eines Interviews oder aber durch eine Mitwirkung an Podiumsdiskussionen erbracht werden. Grafische Leistungen könnten etwa in Form der (Weiter-)Entwicklung kartographischer Materialien, statistischer Darstellungen, grafischer Abbildungen von Prozessen und Kreisläufen, oder aber in der Ableitung von Zusammenhängen oder Sachverhalten aus Bildquellen bestehen. Auf schriftliche und mündliche Leistungen, die in der schulischen Praxis nach wie vor fast ausschließlich zur Leistungsbeurteilung herangezogen werden, wird an dieser Stelle nicht noch einmal ins Detail gegangen.

In diesem Artikel wird ein Vier-Säulenmodell, FO(U)R GW, vorgestellt, im Rahmen dessen Leistungen - entsprechend des Reglements der LBV - in vier Teilbereichen erbracht werden können: So werden praktische, grafische, schriftliche und mündliche Leistungen ermöglicht und gleich gewichtet (Vgl. \$3 Abs. 5 LBV). Das Modell fußt auf der Erlangung so genannter Credits (vgl. 2.2 Definition eines Credits), die für die Erbringung von Teilleistungen in jeder der vier ,Leistungssparten' vergeben werden. Eine Mindestanzahl an zu erreichenden Credits in jeder der vier Dimensionen kann festgelegt werden, um ein Mindestmaß an Mitarbeit der Schüler/innen auch in Säulen, auf welche ihrerseits weniger Gewicht gelegt wird, sicherzustellen.

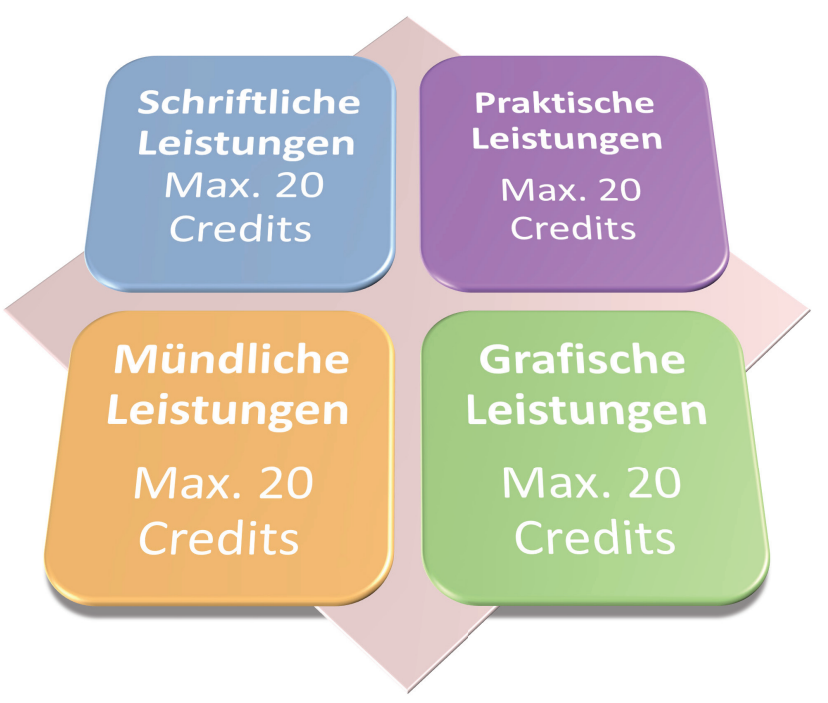

Abb. 1: Übersicht $F O(U) R G W$-Konzept für ein Semester, Quelle: Eigene Darstellung Peter Koller

Im Modell $\mathrm{FO}(\mathrm{U}) \mathrm{R}$ GW sollen Leistungen bewusst auf verschiedenartigen Pfaden dokumentierbar und damit erreichbar sein. Es soll eine Abweichung vom in der Sekundarstufe nach wie vor herrschenden Postulat der Alleinstellung von schriftlichen und mündlichen Leistungen ermöglichen (vgl. Stern 2010, 27). Das Modell ist ganz gezielt auf dem Fundament eines erweiterten, ,breiten' Leistungsbegriffs aufgesetzt, um individuelle Stärken von Schüler/innen besser zur Geltung bringen zu können. Dies wird insbesondere dadurch ermöglicht, dass die Anforderung für eine Beurteilung mit, Sehr gut' bereits mit einem Mindesterwerb von 50 Credits verbunden ist, obwohl insgesamt sogar bis zu 80 Credits im Semester erreichbar sind (siehe Abb. 1 Übersicht $F O(U) R$ GW-Konzept). Diese Untergrenze - deutlich unter dem Maximum an erreichbaren Credits angesetzt ermöglicht es Schüler/innen, sich individuell auf von ihnen selbst festgelegte Wege der Leistungserbringung fokussieren zu können. Ein Mangel an dokumentierten Leistungen in einer Säule kann mit Stärken in einer anderen Säule ausgeglichen werden. Zudem soll durch eine entsprechende, temporäre Taktung der zu erbringenden Teilleistungen im Laufe des Semesters sichergestellt sein, dass die Schüler/innen bereits ab der ersten Unterrichtseinheit Credits in jeder der vier Säulen erlangen können. Somit werden natürliche ,Durchhängephasen' (z. B. emotionale Tiefschläge) wie sie bei jungen Menschen durchaus eintreten können - im FO(U)R GW-Modell durch die gegebene temporäre Flexibilität bei der Leistungserbringung abgefedert. Durch diesen Aspekt sollte auch die Gefahr des bekannten, zum Ende des Semesters beginnenden, ,Bulimie-Lernens' minimiert sein.

Das entwickelte Leistungskonzept ermöglicht also eine auf die Lebensphasen bezogene Leistungserbrin- 
gung mit Erarbeitungsphasen während des gesamten Semesters (siehe Abb. 2). Dieser Gesichtspunkt, in Kombination mit der Berücksichtigung der Individualität von Schüler/innen (welche sich auf ihre Leistungserbringungsarten spezialisieren können), macht es damit besonders ,schüler/innennahe', da es in im Hinblick auf Vorbildung, Migrationshintergrund, Sozialisation im Elternhaus immer heterogener werdenden Klassen eine besondere Berücksichtigung der damit einhergehenden, immer vielfältigeren, Talente ermöglicht. Die Individualisierungskomponente des Modells ermöglicht ferner die Schaffung von individuellen Lernräume und -arrangements für die Schüler/innen. Damit wird eine Basis für einen gelungenen inklusiven Unterricht in Klassen mit hoher Diversität der Schüler/innen gelegt (vgl. Saalfrank 2013). Des Weiteren ermöglicht ein breites ,Leistungsangebot ' für die Schüler/innen auch die Möglichkeit ein breites Spektrum an lebensrelevanten Kompetenzen erwerben zu können.

Das Modell liegt zudem ganz im ,Puls der Zeit', in der traditionell gewachsene eindimensionale Leistungsverständnisse zunehmend hinterfragt werden. So ist erst $2016 \mathrm{im}$ tertiären Bildungsbereich ein neuartiges, auf einem heterogenen Leistungsbegriff aufgebautes, Leistungsbewertungskonzept für AuswahlverfahrenfürProfessor/innenmitdemDiversitätsmanagement-Preis des BMBWF ausgezeichnet worden. ${ }^{1}$

\subsection{Damit einhergehende Ansprüche an die Lehrperson}

Das FO(U)R GW-Konzept stellt hohe Ansprüche an die Lehrperson, erfordert es doch ein entsprechendes Ausmaß an Methodenvielfalt: Ohne ein adäquates Angebot an schüler/innenzentrierten Unterrichtsmethoden und -instrumenten können Leistungsbeurteilungen in den vier heterogenen Leistungsdimensionen (schriftlich, mündlich, grafisch, praktisch) im Rahmen eines Semesters, seitens der Lehrperson, gar nicht angeboten werden. Eine hohe intrinsische Motivation, als auch ein ausreichendes Maß an (fach-)didaktischem Theoriewissen seitens der Lehrkraft, sind daher unabdingbare Voraussetzungen zur Implementierung eines solchen Modells.

Des Weiteren erfordert das FO(U)R GW-Modell jedenfalls einen hohen Organisations- bzw. Administrationsaufwand von Seiten der Lehrkraft. So muss zum einen ein sowohl temporär getaktetes, als auch inhaltlich, im Hinblick auf angebotene Leistungsfor-

\footnotetext{
Vgl. Pressemitteilung der Wirtschaftsuniversität: Leistungsbewertungskonzept ,uLiKe ' der WU mit DiversitätsmanagementPreis ausgezeichnet, online abgerufen am 04.05.2018: https:// www.wu.ac.at/presse/presseaussendungen/presseaussendung-details/detail/leistungsbewertungskonzept-ulike-der-wu-mit-diversitaetsmanagement-preis-ausgezeichnet/.
}

men und Kompetenzen abgestimmtes, ausgeklügeltes Leistungsangebot entwickelt werden. Zum anderen wiederum muss ein auf die jeweiligen individuell gewählten Schwerpunkte der Schüler/innen zugeschnittener Planungsraster zur transparenten Dokumentation der erreichten Leistungen erstellt werden.

\section{Erläuterung wesentlicher Faktoren des Modells}

Im folgenden - eher technisch und theoretisch orientierten - Abschnitt werden wesentliche Rahmenbedingungen (u. a. rechtlicher Art) und Komponenten, die bei geplanter Umsetzung von $\mathrm{FO}(\mathrm{U}) \mathrm{R}$ GW von Lehrpersonen unbedingt zu berücksichtigen sind, aufgezeigt: So wird etwa das Verständnis von Credits im Sinne des FO(U)R GW-Konzepts und deren Verschränkung mit den von Schüler/innen zu erwerbenden Kompetenzen dargelegt. Praktisch näher gebracht wird das FO(U)R GW-Modell dann anhand des Exempels einer 7. Klasse AHS im dritten und letzten Abschnitt (siehe Kapitel 3, insb. Abb. 2 mit einer veranschaulichten Semesterplanung).

\subsection{Anforderung der Erfüllung des, Fachlehr- plans' sowie aller, wesentlichen Bereiche' desselben durch die Schüler/innen}

Nach $₫ 18$ Abs. 1 SchUG idF. BGBl. I Nr. 35/2018 sind als Maßstab für die Leistungsbeurteilung die Forderungen des Lehrplanes heranzuziehen. Gemäß der Lehrplanverordnung idF. BGBl. II Nr. 30/2018 (Dritter Teil, Z1: Unterrichtsplanung für Lehrer/innen) sind in der Oberstufe die inhaltlichen Vorgaben der jeweiligen ,Fachlehrpläne' verbindlich umzusetzen.

In Concreto heißt das, dass im Rahmen des VierSäulenkonzepts jede Schülerin/jeder Schüler trotz der Möglichkeit der Schwerpunktsetzung auf Leistungserbringungsformen für sich den ,Fachlehrplan' in GW erfüllen muss. Die Einführung der Neuen Oberstufe (NOST) bringt mit der verpflichtenden Erfüllung aller wesentlichen Bereiche der lehrplanmäßigen Anforderungen durch die Schüler/innen eine weitere Anforderung. ${ }^{2}$

Vgl. dazu die Regelung in $\$ 14$ Abs. 5 LBV idF. BGBl. II Nr. 424/2016. Wonach Leistungen mit "Genügend“ zu beurteilen sind, „(...) mit denen der Schüler die nach Maßgabe des Lehrplanes gestellten Anforderungen in der Erfassung und in der Anwendung des Lehrstoffes sowie in der Durchführung der Aufgaben in den wesentlichen Bereichen überwiegend erfüllt. "Zum konkreten Herunterbrechen dieser wesentlichen Bereiche auf die konkreten Lehrpläne für Geographie und Wirtschaftskunde in der Sekundarstufe II siehe die Publikation von Hinsch et. al (2017: 80-84) in GWUnterricht 148. 
Tab. 1: Arbeitsaufwand pro Credit und hypothetischer Gesamtaufwand zur Erreichung eines, Sehr gut; Quelle: Eigene Darstellung Peter Koller

\begin{tabular}{|c|c|c|c|c|c|c|}
$\begin{array}{c}\text { Grundsätzli- } \\
\text { cher Arbeits- } \\
\text { aufwand in } \\
\begin{array}{c}\text { Minuten pro } \\
\text { Credit }\end{array}\end{array}$ & $\begin{array}{c}\text { Qualität des } \\
\text { erzeugten } \\
\text { Produkts }\end{array}$ & Multiplikator & $\begin{array}{c}\text { Tatsächlicher } \\
\text { Arbeitsauf- } \\
\text { wand in } \\
\text { Minuten pro } \\
\text { Credit }\end{array}$ & $\begin{array}{c}\text { Wochenanzahl } \\
\text { Semester }\end{array}$ & $\begin{array}{c}\text { Tatsächlicher } \\
\text { Creditanzahl } \\
\text { für ,Sehr gut' }\end{array}$ & $\begin{array}{c}\text { Gesamtar- } \\
\text { beitsaufwand } \\
\text { pro Semester/ } \\
\text { Stunden }\end{array}$ \\
\hline $30^{\prime}$ & sehr gut & 1 & $30^{\prime}$ & 15 & 50 & $25 \mathrm{~h}$ \\
\hline $30^{\prime}$ & gut & 0,8 & $37,5^{\prime}$ & 15 & 50 & $31,25 \mathrm{~h}$ \\
\hline $30^{\prime}$ & zufriedenstellend & 0,6 & $50^{\prime}$ & 15 & 50 & $41,67 \mathrm{~h}$ \\
\hline $30^{\prime}$ & ausreichend & 0,5 & $60^{\prime}$ & 15 & 50 & $50 \mathrm{~h}$ \\
\hline
\end{tabular}

Ein gewisser Spielraum bleibt der Lehrperson jedoch, da eine flexible Unterrichtsgestaltung durch die eingeräumte Möglichkeit der zeitlichen Gewichtung und der konkreten Umsetzung der Vorgaben durch die Lehrkraft möglich ist. ${ }^{3}$

Dem entsprechend ist auch im Rahmen des vorgelegten Modells die Möglichkeit zu schaffen, dass die Lehrplanziele auch mit Leistungen, die sich auf zwei bzw. drei der vier Säulen fokussieren, abarbeitbar sind. Dies bedeutet nichts anderes, als dass jede der vier Säulen der Leistungserbringung in sich möglichst alle wesentlichen Bereiche des Lehrplans abdecken soll.

\subsection{Definition eines Credits}

Credit ist im Sinne des Verfassers am ehesten mit dem Terminus, Guthaben' übersetzbar: Die Bezeichnung wurde bewusst an die für die Darstellungen von Leistungen an Hochschulen im Rahmen des Bologna-Prozesses entwickelte Konzeption der ECTS (European Credit Transfer System) angelehnt. Allerdings zielt ein Credit im Rahmen des FO(U)R GW-Konzepts nicht nur auf den zu erwartenden Arbeitsaufwand inklusive etwaiger Vor- und Nachbereitungstätigkeit, sondern letztlich auch auf die Qualität des vorgelegten Endproduktes ab. Die Qualität des erzeugten Produktes fließt als Multiplikator in die Errechnung ein. Eine ,sehr gute' Qualität entspricht einem Multiplikator von 1, eine, gute' Qualität einem von 0,8, eine ,zufriedenstellende' Qualität einem von 0,6 eine ,ausreichende` Qualität einem von 0,5 . Sollte die Qualität

\footnotetext{
Siehe Verordnung des Bundesministers für Unterricht und Kunst vom 14. November 1984 über die Lehrpläne der allgemeinbildenden höheren Schulen; Bekanntmachung der Lehrpläne für den Religionsunterricht an diesen Schulen idF. BGBl. II Nr. 30/2018, Dritter Teil, Z1 Unterrichtsplanung der Lehrer/ innen: „Die Vorgaben (Lehrziele, Themenbereiche usw.) im Abschnitt "Lehrstoff" der einzelnen Unterrichtsgegenstände der Oberstufe sind verbindlich umzusetzen; dies gilt auch für den Fall schulautonomer Stundenreduktionen. Die zeitliche Gewichtung und die konkrete Umsetzung der Vorgaben obliegen den Lehrerinnen und Lehrern und ermöglichen somit eine flexible Unterrichtsgestaltung (...)."
}

,nicht ausreichend' sein, werden für die entsprechende Leistung auch keine Credits am Leistungskonto der Schüler/innen gutgeschrieben.

Ein erworbener Credit entspricht, bei bestmöglicher Bewertung des vorgelegten Endprodukts, einem geschätzten Arbeitsaufwand von 30 Minuten. Anzumerken ist, dass der Aufwand für einzelne Schüler/innen natürlich variieren kann und nicht immer dem kalkulierten Durchschnittsmaß von $30 \mathrm{Minu}-$ ten entsprechen wird. Für eine Bewertung mit ,Sehr gut $^{\text {' }}$ - welche 50 erworbene Credits erfordert - ist dementsprechend bei bestmöglicher Bewertung der vorgelegten ,Endprodukte' ein Arbeitsaufwand von rund 25 Stunden - was in einem Semester mit 15 Wochen einem wöchentlichen Arbeitsaufwand von ca. einer Stunde und 40 Minuten entspricht - zu kalkulieren ${ }^{4}$. Dieser Arbeitsaufwand kann zu großen Teilen auch bereits während der zwei Mal wöchentlich stattfindenden Präsenzeinheiten $(2$ x 50 Minuten) bewältigt werden. Je nach gelieferter Qualität der ,Endprodukte ist auch ein deutlich höherer vorgesehener Arbeitsaufwand für ein ,Sehr gut' möglich (theoretisch sind bis zu 50 Stunden und somit maximal knapp über drei Stunden Gesamtarbeitsaufwand pro Woche möglich, hier ist allerdings wieder zu berücksichtigen, dass große Teile dieses Aufwands während der Präsenzeinheiten bewältigt werden können, vgl. Tab. 1).

Durch die Noten ist die Selbstständigkeit der Arbeit, die Erfassung und die Anwendung des Lehrstoffes, die Durchführung der Aufgaben und die Eigenständigkeit der Schüler/innen zu beurteilen ( $\$ 18$ Abs. 3 SchUG). Gemäß der Leistungsbeurteilungsverordnung (LBV) ist ein ,Sehr gut' nur für Leistungen, mit denen der Schüler/die Schülerin die Anforderungen des Lehrplans "weit über das Wesentliche hinausgehendem Ausmaß" erfüllt und "deutliche Eigenständigkeit beziehungsweise Fähigkeit zur selbstständigen Anwen-

2 Wochenstunden laut Stundentafel des Lehrplans der Bildungsanstalt für Sozialpädagogik im IV. Jahrgang; Zweites Semester ca. 15 Wochen, erstes Semester ca. 19 Wochen. 
dung seines Wissens und Könnens zeigt", zu vergeben (\$ 14 Abs. 2 LBV idF. BGBl. II Nr. 424/2016). Von Schüler/innen die ein ,Sehr gut' anstreben sind daher Kompetenzen in allen Anforderungsbereichen (I ,Reproduktion', II ,Reorganisation und Transfer', sowie III ,Reflexion und Problemlösung - siehe Sitte 2011, $\left.39-41^{5}\right)$ und insbesondere auf Kompetenzniveau III, ,Reflexion und Problemlösung', nachzuweisen.

Für das $\mathrm{FO}(\mathrm{U}) \mathrm{R}$ GW-Konzept bringt dies eine weitere Anforderung mit sich, müssen die für ein ,Sehr gut' zu sammelnden 50 Credits doch auch entsprechende Leistungsnachweise in den höheren Kompetenzniveaus II und III enthalten.

Dementsprechend wird nach drei Arten von Credits differenziert: I-Credits, II-Credits sowie III-Credits. Grundsätzlich unterscheiden sich die Credits in ihrer Wertigkeit nicht, jedoch wird etwa ein ,Sehr gut nur vergeben, wenn zumindest jeweils 15 II-Credits bzw. 15 III-Credits unter den gesammelten 50 Credits sind.

$\mathrm{Ab}$ einem ,Befriedigend', wo zwar „merkliche Ansätze an Eigenständigkeit"erkennbar sein müssen, aber keine Fähigkeit zur Anwendung des Wissens und Könnens auf neuartige Aufgaben mehr erforderlich ist, ist es meiner Meinung nach Auslegungssache der Lehrperson, ob Leistungen im Bereich des Kompetenzniveaus III noch erbracht werden müssen.

Im vorliegenden Modell werden bis hin zu einem ,Genügend' (wenn auch dort nur einer, je wesentlichem Lehrplan-Bereich) Credits im Bereich ,Reflexion und Problemlösung' gefordert, erscheint es doch fraglich, wie ein ,wesentlicher' Bereich des Lehrplans von einer Schülerin/einem Schüler überwiegend erfüllt werden soll, wenn eine Nullleistung im Bereich ,Reflexion und Problemlösung' gegeben ist.

Wie genau die Erfüllung, wesentlicher' Lehrplanbereiche mit der Verarbeitungstiefe der Inhalte bei Schülerinnen und Schülern (Kompetenzniveaus I-III) zusammenspielt ist derzeit noch offen. Es handelt sich aber um eine zentrale Fragestellung, die in naher $\mathrm{Zu}-$ kunft zu klären und zu regeln sein wird.

\subsection{Klassifizierung der Credits}

\begin{tabular}{|ll}
$\square \quad$ I-Credit & $\begin{array}{l}\text { Credit im Kompetenzniveau I: } \\
\text {,Reproduktion' }\end{array}$ \\
II-Credit & $\begin{array}{l}\text { Credit im Kompetenzniveau II: } \\
\text {,Reorganisation und Transfer' }\end{array}$ \\
III-Credit & $\begin{array}{l}\text { Credit im Kompetenzniveau III: } \\
\text {,Reflexion und Problemlösung' }\end{array}$
\end{tabular}

\footnotetext{
Anforderungsbereiche auch wie folgt darstellbar: I: Wiederholung, II: Umorganisation von Wissen bzw. Transfer, III: komplexe Anwendung und Transfer, echte Problemlösung (Bundesministerium für Bildung und Frauen 2012, 13)
}

\subsection{Bewertungssystem}

\begin{tabular}{|c|c|c|c|}
\hline \multirow{4}{*}{ Sehr gut } & Insgesamt & Mind. $50 x$ & Credits \\
\hline & ... davon & Mind. $15 x$ & II-Credit \\
\hline & ... davon & Mind. $15 x$ & III-Credit \\
\hline & ... sowie & \multicolumn{2}{|c|}{$\begin{array}{l}\text { Alle, wesentlichen' Bereiche des Lehrplans } \\
\text { vollständig erfüllt, darüber hinausgehende } \\
\text { Bereiche vollständig erfüllt. }\end{array}$} \\
\hline \multirow{4}{*}{ Gut } & Insgesamt & Mind. $42 x$ & Credits \\
\hline & ... davon & Mind. $12 x$ & II-Credit \\
\hline & ... davon & Mind. 10x & III-Credit \\
\hline & ... sowie & \multicolumn{2}{|c|}{$\begin{array}{l}\text { Alle, wesentlichen' Bereiche des Lehrplans } \\
\text { vollständig erfüllt, darüber hinausgehende } \\
\text { Bereiche überwiegend erfüllt. }\end{array}$} \\
\hline \multirow{4}{*}{$\begin{array}{c}\text { Befriedi- } \\
\text { gend }\end{array}$} & Insgesamt & Mind. $35 x$ & Credits \\
\hline & ... davon & Mind. 10x & II-Credit \\
\hline & ... davon & Mind. $5 x$ & III-Credit \\
\hline & ... sowie & \multicolumn{2}{|c|}{$\begin{array}{l}\text { Alle, wesentlichen' Bereiche des Lehrplans } \\
\text { vollständig erfüllt. }\end{array}$} \\
\hline \multirow{4}{*}{$\begin{array}{l}\text { Genü- } \\
\text { gend }\end{array}$} & Insgesamt & Mind. $20 x$ & Credits \\
\hline & ... davon & Mind. $5 x$ & II-Credit \\
\hline & ... davon & Mind. $3 x$ & III-Credit \\
\hline & ... sowie & \multicolumn{2}{|c|}{$\begin{array}{l}\text { Alle, wesentlichen' Bereiche des Lehrplans } \\
\text { überwiegend erfüllt. }\end{array}$} \\
\hline
\end{tabular}

\subsection{Transparenz für die Schüler/innen}

Zahlreiche empirische Erkenntnisse (u.a. Helmke 2007) belegen, dass Transparenz hinsichtlich des Erwartungshorizonts und damit verknüpft auch hinsichtlich der Leistungsfeststellung entscheidend für eine erfolgreiche Durchführung von kompetenzorientiertem Unterricht ist. Da FO(U)R GW doch einen gewissen Komplexitätsgrad aufweist, ist es für eine erfolgreiche Umsetzung des Modells essenziell, von Beginn an bei Schüler/innen Transparenz im Hinblick auf die Leistungsanforderungen sowie die Lernziele zu schaffen (vgl. Stern 2010, 43). Dementsprechend ist das Modell bereits zu Beginn des Schuljahres detailliert vorzustellen. Ein Handout, auf dem Modell, Creditdefinition, Creditarten, Bewertungssystem sowie die konkreten Möglichkeiten während des Semesters Credits zu sammeln, erläutert werden, ist dabei auszugeben. Damit soll bei den Schüler/innen Verständnis für das System $\mathrm{FO}(\mathrm{U}) \mathrm{R}$ GW und der damit verbundenen individuellen Anforderungen geweckt werden.

Als Aufgabe ist den Schüler/innen mitzugeben, bis zur darauffolgenden Unterrichtseinheit eine grundlegende Vorstellung auf den für sie ,stimmigen' Korb an Leistungsdimensionen und darin enthaltenen Teilleistungen zu entwickeln und diese der Lehrperson mitzuteilen. 
Auf dieser Basis ist in der folgenden Einheit eine individuelle ,Leistungsvereinbarung' zwischen Lehrperson und Schüler/in zu schließen. Die Inhalte derselben sollen sicherstellen, dass zum Ende des Semesters die wesentlichen Lehrplanbereiche individuell abgedeckt sind und bei stetig, sehr guter' Leistungsqualität von jeder Schülerin bzw. jedem Schüler bis dorthin auch zumindest 50 Credits erreicht werden können. Während des Semesters sind bei Bedarf von Schüler/innen, in Hinblick auf die Lebensphasenund Interessensbezogenheit des Modells, Abänderungen der Vereinbarung möglich. Die Lehrkraft hat die individuell ausverhandelten Leistungsvereinbarungen in eine möglichst elaborierte Excel-Datenbank zu übertragen und erbrachte Credits darin während des Semesters kontinuierlich hinsichtlich der jeweiligen Leistungen der Schüler/innen zu verzeichnen.

Dies erlaubt der Lehrperson individuelle Lernfortschritte jederzeit im Auge zu behalten, was wiederum fundierte Feedbackgespräche mit Schüler/innen als auch mit Elternteilen während des Semesters ermöglicht. $\mathrm{Zu}$ guter Letzt ist diese Form der Dokumentation auch ein hervorragendes Fundament für ein ausführliches, endsemestrig, neben der Notenbeurteilung ausgestelltes, ,Fortschrittsgutachten' der Lehrperson im Hinblick auf die individuelle Entwicklung von Kompetenzen der Schüler/innen (vgl. Stern 2010, 86).

\section{Exempel der praktischen Umsetzung des Modells}

\subsection{Exemplarische Aufbereitung von FO(U)R GW anhand des Lehrplans für das 2. Semester der 7. Klasse AHS}

Illustriert werden soll das Vier-Säulen-Konzept anhand des GW-Fachlehrplans des 2. Semesters der 7. Klasse AHS. Exemplarisch wird das für ein Vier-SäulenKonzept erforderliche Leistungsangebot der Lehrkraft für ein Semester dargestellt, um das Konzept praktisch näher bringen zu können (siehe $A b b$. 2: Planungsraster, gleichzeitig auch Kernstück dieses Kapitels).

Man nehme an, die Lehrperson in einer 7. Klasse AHS im 2. Semester bringt ihren Schüler/innen mittels vielfältiger (auch schüler/innenzentrierter) Unterrichtsmethoden, -formen und -techniken folgende Themenbereiche näher: „Naturräumliche Chancen und Risiken erörtern“, „Demografische Entwicklung und gesellschaftspolitische Implikationen beurteilen", sowie „Unternehmen und Berufsfelder analysieren“. ${ }^{\circ}$

\footnotetext{
6 Verordnung für Lehrpläne - allgemeinbildende höhere Schulen: Lehrplan Geographie und Wirtschaftskunde, 7. Klasse idF. BGBl. II Nr. 230/2018.
}

Diese Inhalte und die ihnen zuträglichen Lernziele müssen individuell von jeder Schülerin und jedem Schüler dieser 7 . Klasse grundlegend erfüllt werden, wenngleich der Lehrperson sehr wohl ein Flexibilitätsspielraum im Hinblick auf die jeweilige Gewichtung einzelner Lernziele bleibt. (siehe Kap. 2.1 $)^{7}$

Auch die grundlegenden methodischen und fachspezifischen Kompetenzen, sowie die Ermöglichung der Darlegung von Leistungen auf unterschiedlichen Kompetenzniveaus, sollen für jede Schülerin und jeden Schüler individuell sichergestellt werden. Darüber hinaus ist mit der Einführung der NOST auch die Anforderung der Erfüllung aller wesentlichen Bereiche der lehrplanmäßigen Anforderungen für die Schüler/innen einhergegangen. Für das 2. Semester der 7. Klasse AHS identifizierten Hinsch, Pichler und Jekel (vgl. Hinsch et al. 2017, 80 ff.) eben jene obig genannten Lernziele auch als die drei wesentlichen Bereiche, die für sich erfüllt werden müssen, um einer negativen Semester-Beurteilung zu entgehen.

\subsection{Planungsraster}

Eine Voraussetzung zur Durchführung bildet ein vor Beginn des Semesters zu erstellender klarer und für die Schüler/innen transparenter Planungsraster. In diesem sind die Teilleistungen, die während des Semesters angeboten und für die Credits entsprechend vergeben werden, jeweils einer der vier Säulen (praktisch, grafisch, schriftlich und mündlich) im Sinne eines Überwiegungsprinzips zugeordnet (Leistungen, die mehrere Dimensionen betreffen und somit transdimensionale Aspekte aufweisen werden so zur Vereinfachung der Planbarkeit für die Lehrperson, sowie für die Schüler/innen, einer der Säulen zugerechnet).

\section{3 Übersicht Teilleistungen am Beispiel der siebenten Klasse AHS (2. Semester)}

Portfolio (16 Credits gesamt): Beim Portfolio werden die schriftlichen Arbeitsleistungen während des Semesters gesammelt und in einem gebündelten Endprodukt zusammengestellt. Dabei können ausgewählte Arbeitsaufträge wahlweise in schriftlicher und/oder grafischer Form aufbereitet werden. Zusätzlich ist dem Portfolio eine Reflexion der individuellen Entwicklung während des Semesters anzufügen, in der die persönlichen Lernfortschritte genannt werden sollen.

Schriftlicher Test (7 Credits): Der Test ist auf freiwilliger Basis und wird schriftlich durchgeführt. Es können aus fünf Fragestellungen vier ausgewählt

Die Anforderung der Erfüllung des ,Fachlehrplans' durch alle Schüler/innen ist aber zu berücksichtigen. 


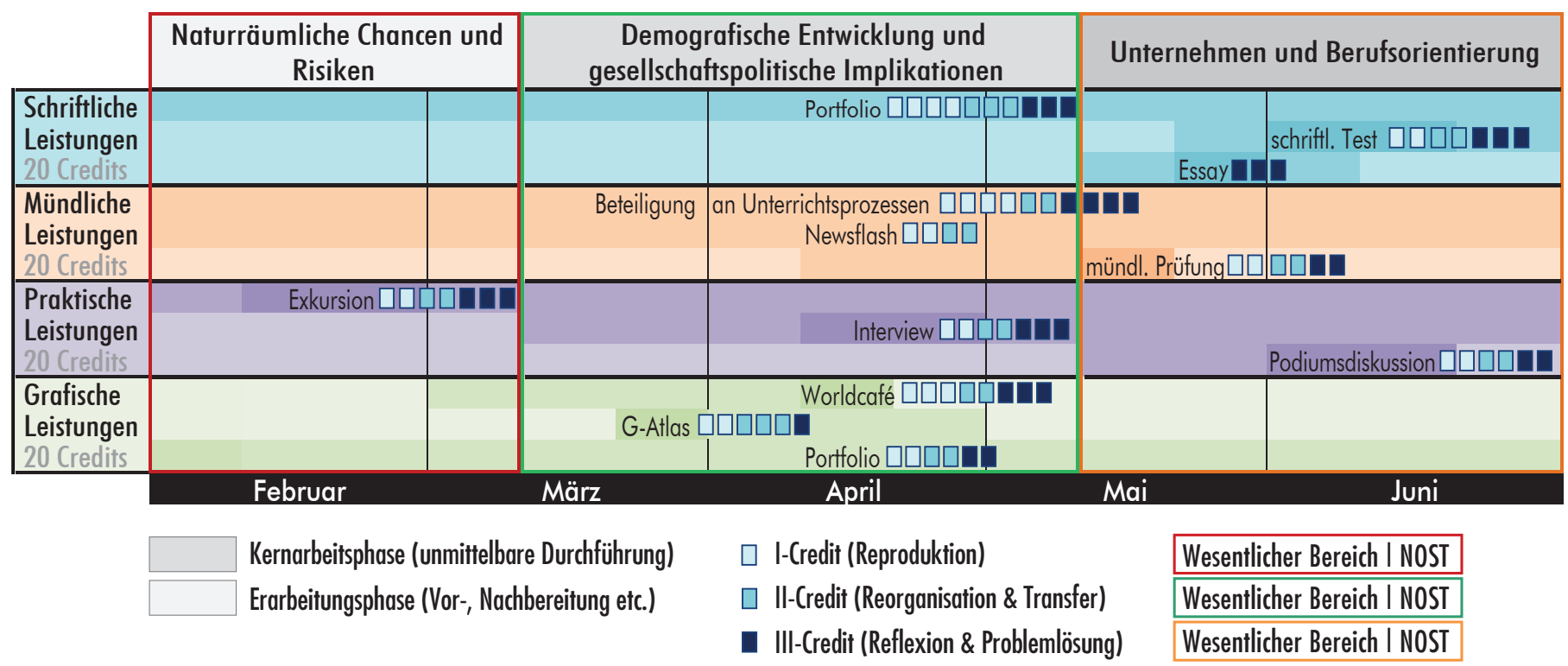

Abb. 2: Semesterplanung nach $F O(U) R$ GW-Modell auf Basis der 7. Klasse AHS; Quelle: Eigene Darstellung Peter Koller

und beantwortet werden. Die Fragen sind so aufgebaut, dass sie mittels geeigneter Operatoren jeweils die drei Kompetenz-Anforderungsbereiche (I: ,Reproduktion', II: ,Transfer', III: ,Reflexion/ Problemlösung') abdecken. Der Test kann nach Rückgabe mithilfe des Lehrbuches und weiterer Materialien noch einmal verbessert und in schriftlich beantworteter Form dem Portfolio angefügt werden.

Mündliche Prüfung (6 Credits): Zwei ausführliche und (inhaltlich) möglichst voneinander unabhängige Fragen werden gestellt. Die Fragen sollen alle drei Kompetenz-Anforderungsbereiche (I: ,Reproduktion', II: ,Transfer', III: ,Reflexion/ Problemlösung') beinhalten. Innovative Elemente, wie die Möglichkeit der einminütigen Einbeziehung einer Mitschülerin bzw. eines Mitschülers, als ,Diskussionsjoker', in den Überlegungsprozess bei einer herausfordernden Fragestellung, sollen integriert werden. Beantwortet muss die jeweilige Fragestellung anschließend jedoch von der Schülerin bzw. dem Schüler, die/ der die mündliche Prüfung ablegt (Adressat/in der Frage), werden.

Essay (3 Credits): Inhaltlich ist die Thematik, Von der Idee hin zum Unternehmen in Österreich abzuhandeln. Schwerpunkte wie z. B. die Hürden, die es bis hin zur Unternehmensgründung zu überwinden gibt, öffentliche Unterstützungen auf diesem Weg bzw. eine Spezialisierung auf eine zeitliche Phase des Gründungsprozesses, können gesetzt werden. Als Basis dafür können eine Befragung von Selbstständigen oder Unternehmer/innen im Bekanntenkreis, sowie eine Internet-Recherche dienen. Die Websites bzw. die Online-Artikel, auf die Bezug genommen wird, sind als Quellen anzugeben.

Beteiligung an Unterrichtsprozessen (10 Credits): Diese setzt sich aus folgenden Teilkomponenten zusammen:

Beteiligung am Lehrer/innen- \& Schüler/innenDialog: Wichtig ist hierbei die Aktivität der Schüler/innen. Sie sollen Interesse zeigen, kritische Fragen stellen, und bei von der Lehrperson aufgeworfenen Fragen aktiv antworten. Erworbene Kompetenzen der Schüler/innen sollen aus den Antworten erkennbar werden.

Beteiligung/Interaktion in Phasen des kooperativen oder offenen Lernens: Hier sind etwa Prozessorganisations- und Kooperationsfähigkeiten von Schüler/innen wichtig. Der Aufmerksamkeitsgrad (Fokus auf die vorgegebene inhaltliche Thematik) und der Grad des „Forschungsantriebs“ von Schüler/innen spielen eine wesentliche Rolle.

Newsflash (4 Credits - Exempel mit Anforderungen zur Creditvergabe): Die Erkenntnisse aus der vorangegangenen Unterrichtseinheit sind methodisch innovativ (Partner/innenarbeit möglich) den Mitschüler/innen in Erinnerung zu rufen. Es können etwa neue und interaktive digitale Tools wie Mentimeter zum Einsatz kommen, um die gesamte Klasse gut einbeziehen und damit aktivieren zu können. Um auch den Anforderungsbereich II, ,Reorganisation und Transfer', bespielen zu können, soll den Schüler/innen die Auswertung wirtschaftlicher oder politischer Sachverhalte aus Medientexten nahe gelegt werden, welche dann für die Klasse aufbereitet wird ( -5 Minuten). 
Creditvergabe:

- 2 x Credits im Bereich I: ,Reproduktion':

- Die Schüler/innen können Inhalte der vergangenen Unterrichtseinheit (Fachbegriffe, Daten, Fakten, Modelle) in komprimierter Form darstellen.

- Wesentliche, in der vergangenen Einheit erarbeitete, Inhalte oder Zusammenhänge können in adäquater Fachsprache und in logischer Struktur wiedergegeben werden.

- 2 x Credits im Bereich II: ,Transfer':

- Eine Einpflege der Inhalte der vergangenen Einheit in einen größeren Zusammenhang soll den Schüler/innen durch eigenes Wissen gelingen.

- Es muss erkenntlich sein, dass die Schülerinnen oder der Schüler einen gesamtheitlichen Zusammenhang der Inhalte der vergangenen Einheit erkannt haben und damit Sinnzusammenhänge zwischen unterschiedlichen Materialien herstellen können.

- Die Schüler/innen können demonstrieren, dass sie gelernte Inhalte auch auf mediale Sachverhalte anwenden können und geographische Sachverhalte aus Medien entnehmen und mit den behandelten Inhalten in Verbindung setzen können.

- Die Schüler/innen können behandelte Inhalte auch in anderen digitalen Methoden wie zum Beispiel Mentimeter anwenden.

Exkursion ( 7 Credits): Die Exkursion wird in diesem Beispiel, zweitägig, im Biosphärenpark großes Walsertal, in Vorarlberg durchgeführt. Anhand dieses Beispiels sollen die naturräumlichen Voraussetzungen sowie politische und gesellschaftliche Interessen als Ursachen ökologischer Probleme erkannt werden. Das Biosphärenparkkonzept soll als Lösungskonzept im Hinblick auf ökologisch nachhaltige Bewirtschaftung eines Lebensraums in Vor- und Nachteilen analysiert werden. In der Vorbereitungsphase sollen gezielte Fragen an Bürgermeister/innen, Landwirt/innen sowie Gewerbetreibende oder allgemein an Bewohner/ innen im Biosphärenpark erstellt werden. Auf Basis der Befragungen soll die kritische Reflexion des Biosphärenparkkonzepts durchgeführt werden.

Interview (7 Credits): Die Lebenssituation ausgewählter Bevölkerungsgruppen ist vor dem Hintergrund des Phänomens „Fremdsein“ zu analysieren: Ein Interview mit einer Person mit Migrationshintergrund ist durchzuführen, wofür vorbereitend ein Interviewleitfaden erstellt wird. Anschließend folgt eine kritische Reflexion des Interviews im Hinblick auf die erhaltenen Antworten, aber auch die eigene Rolle als interview- ende Person. Danach erfolgt anhand einer Literaturrecherche (Zeitung o. ä.) eine Einbettung der erhaltenen Antworten in Bezug auf die Gesamtsituation dieser Personengruppe in Österreich. Es sollen auch anhand der Gesamtarbeit Herausforderungen einer multikulturellen Gesellschaft identifiziert und dargelegt werden.

Podiumsdiskussion (6 Credits): En dem vorgestellten Beispiel wird eine moderierte Podiumsdiskussion zum Thema ,Pressekonferenz zum Börsengang eines Unternehmens' durchgeführt. Die jeweiligen Rollen sind detailliert vorzubereiten: Vorstandsvorsitzende/r, Marketingleiter/in, Journalist/in, Moderator/in, potenzielle Aktionäre etc.

World-Café (8 Credits): Die Basis der Stationen werden grafische Aufbereitungen in Form von Plakaten zur demografischen Entwicklung und deren gesellschaftliche Implikationen für Österreich bilden, so sind beispielsweise Bevölkerungspyramiden, kartographische Produkte, Bevölkerungsstatistiken - immer in Verknüpfung mit relevanten Ereignissen - zu erstellen. Eine Reflexion des World-Café in Bezug auf den eingeschätzten Erfolg bei der Umsetzung und Vermittlung der Inhalte ist dem Portfolio hinzuzufügen.

Gender-Atlas (6 Credits): Das Projekt,Gender-Atlas für die Schule' des Instituts für Geographie und Regionalforschung der Universität Wien (http:// genderatlas.at/schule/) wird in der Klasse vorgestellt. Die Bedeutung einer kartografischen Aufarbeitung der politischen und gesellschaftlichen Situation für Frauen in Österreich soll den Schüler/innen nähergebracht werden. Die jeweiligen kartographischen Darstellungen sind zu präsentieren und in ihrer Semantik den Schüler/innen näherzubringen. Zudem soll zukünftiges Potenzial solcher kartografischer Aufbereitungen aufgezeigt und diskutiert werden.

\section{$4 \quad$ Fazit}

Es zeigte sich im Zuge der Entwicklung des FO(U)R GW-Konzepts wie vielschichtig und komplex die Vorgaben zur Leistungsbeurteilung sind. Dementsprechend herausfordernd war es, all diese unbedingt zu berücksichtigenden Aspekte als Rahmenbedingungen in das vorliegende Konzept einzubeziehen.

„Ich hätte da noch eine Frage ... "- dieses geflügelte Zitat des knautschigen Inspektors Columbo bringt die wesentliche Prämisse des vorlegten 4-Säulenmodells FO(U)R-GW auf den Punkt: Mittels multimethodischem und schüler/innenzentriertem Unterricht, der vier Leistungsarten (mündlich, schriftlich, grafisch, praktisch - jeweils gleich gewichtet) ermöglicht, wird 
metaphorisch eine ganze Palette an ,Fragen' an die Schüler/innen gerichtet. Nämlich, inhärente Fragen bezüglich ihrer Mitwirkung und solche ihrer Einbringung von Potenzialen und Kapazitäten (Kompetenzen) in unterschiedlichsten Unterrichtssituationen, -prozessen und -settings. Damit wird eine Dokumentation unterschiedlichster Entwicklungsspuren oder Entwicklungswege von Schüler/innen und daraus folgend eine adäquatere Leistungsbeurteilung ermöglicht.

Herausgekommen ist ein in der Theorie durchaus verheißungsvolles Modell, dessen praktische Erprobung aber noch aussteht. Es bleibt zu hoffen, dass FO(U)R GW tatsächlich in die Praxis und damit in die Schulen gelangt und sich dort auch vor Ort bewährt: Zum einen im Sinne einer die Inklusion fördernden Individualisierung für die Schüler/innen, die ihre heterogenen Talente nach dem Motto ,Stärken stärken' besser in das Unterrichtsgeschehen bzw. Unterrichtsprozesse einbringen können und damit hoffentlich vermehrt intrinsische Motivation zur Auseinandersetzung mit Lehrinhalten entwickeln. Zum anderen aber auch für Lehrer/innen, da ein Einsatz einen Antrieb zur Verbesserung des Selbstorganisationsgrades sowie der Weiterentwicklung des eigenen methodischen Repertoires und der eigenen didaktischen Kompetenzen darstellen könnte.

\section{Literatur}

Bundesminister für Unterricht und Kunst (1984): Verordnung über die Lehrpläne der allgemeinbildenden höheren Schulen; Bekanntmachung der Lehrpläne für den ReligionsunterrichtandiesenSchulenidF. BGBl.IINr. 111/2017.

Bundesminister für Unterricht und Kunst (1974): Verordnung über die Leistungsbeurteilung in Pflichtschulen sowie mittleren und höheren Schulen (Leistungsbeurteilungsverordnung), idF. BGBl. II Nr. 424/2016.

Bundesgesetz über die Ordnung von Unterricht und Erziehung in den im Schulorganisationsgesetz geregelten Schulen (Schulunterrichtsgesetz - SchUG) idF. BGBl. I Nr. 120/2016.
Bundesministerium für Bildung und Frauen (2012): Geographie und Wirtschaftskunde. Die kompetenzorientierte Reifeprüfung. Richtlinien und Beispiele für Themenpool und Prüfungsaufgaben.

Bundesministerium für Unterricht, Kunst und Kultur (2007): Informationsblätter zum Schulrecht Teil 3: Leistungsfeststellung und Leistungsbeurteilung.

Helmke, A. (2007): Lernprozesse anregen und steuern. Was wissen wir über Klarheit und Strukturiertheit? Pädagogik, 59(6), S. 44-47.

Hinsch, S.; H. Pichler \& T. Jekel (2017): Wesentliche Bereiche des Lehrplans Geographie und Wirtschaftskunde als Beurteilungsgrundlage. In: GW-Unterricht 148, S. 80-84.

Institut für Geographie und Regionalforschung der Universität Wien (2017): GenderATLAS für die Schule.,URL: http://genderatlas.at/schule/ (18.04.2018).

Sitte, C. (2011): Maturafragen NEU (!?) - eine schrittweise Annäherung an eine kompetenzorientierte Form in Geographie und Wirtschaftskunde. In: GW-Unterricht 123, S. 24-41.

Stern, T. (2010): Förderliche Leistungsbewertung. Wien. Österreichisches Zentrum für Persönlichkeitsbildung und soziales Lernen, im Auftrag des Bundesministeriums für Unterricht, Kunst und Kultur, Wien.

Verheyen, N. (2018): Liebe, Gehorsam oder Großes leisten? Leistungssemantiken im 19. Jahrhundert zwischen sozialer Verpflichtung und individuellem Können. In: Reh S. \& N. Ricken (Hrsg.): Leistung als Paradigma. Zur Entstehung und Transformation eines pädagogischen Konzepts. Wiesbaden: Springer VS, S. 165-189.

Wirtschaftsuniversität Wien (2017): Leistungsbewertungskonzept „uLiKe“ der WU mit DiversitätsmanagementPreis ausgezeichnet. URL: https://www.wu.ac.at/presse/ presseaussendungen/presseaussendung-details/detail/ leistungsbewertungskonzept-ulike-der-wu-mit-diversitaetsmanagement-preis-ausgezeichnet/ (04.05.2018).

Wolf-Thorsten S. (2013): Inklusive Bildung im Kontext von Modellen guten Unterrichts. In: Zeitschrift für Inklusion-online.net. URL: https:/www.inklusion-online.net/ index.php/inklusion-online/article/view/18 (20.07.18). 